\title{
Unmasking of Occult Myasthenia Gravis in Hypertrophic Cardiomyopathy
} by Beta Blockers

\section{Srivatsa Nadig ${ }^{1}$, Aditya Kapoor ${ }^{1 *}$, Ritesh Agrawal ${ }^{2}$ and Anjali Mishra ${ }^{2}$}

${ }^{1}$ Department of Cardiology, Sanjay Gandhi Postgraduate Institute of Medical Sciences, India

${ }^{2}$ Department of Endocrine Surgery, Sanjay Gandhi Postgraduate Institute of Medical Sciences, India

\begin{abstract}
Association of Hypertrophic cardiomyopathy $(\mathrm{HCM})$ with immune linked disorders like myasthenia gravis is extremely rare. Various pharmacologic agents can potentially precipitate or unmask underlying signs and symptoms of myasthenia gravis. Oral beta blockers are an important mainstay in the pharmacological management of patients with $\mathrm{HCM}$. We report a case of obstructive $\mathrm{HCM}$ who when treated with beta blockers developed increasingly severe weakness and fatigue. Detailed clinical examination led to a clinical diagnosis of underlying myasthenia gravis which was secondary to a thymoma. The patient had developed acute unmasking of the latent myasthenia gravis following institution of therapy with beta-blockers. The patient underwent successful resection of the thymic mass and made an uneventful recovery. Symptoms like fatigue, shortness of breath and weakness are common among patients with $\mathrm{HCM}$ and are also frequent in patients with myasthenia gravis, sometimes making a clinical diagnosis difficult. It is important for physicians to be aware of such unusual associations to be able to appropriately manage such cases in clinical practice.
\end{abstract}

Keywords: Hypertrophic Cardiomyopathy (HCM); Thymoma; Sarcomeric proteins

\section{Introduction}

Hypertrophic cardiomyopathy (HCM) is a genetic disorder that has a primarily autosomal dominant inheritance pattern, with variable penetrance and phenotypic expressivity [1]. Although its etiology has been linked to defects in various genes encoding for the sarcomeric proteins, association of HCM with immune linked disorders like myasthenia gravis is extremely rare. We report a case of obstructive HCM who when treated with beta blockers developed acute unmasking of latent myasthenia gravis, which was secondary to an underlying thymoma. The patient underwent successful resection of the thymic mass and made an uneventful recovery. Physicians need to be aware of such unusual associations to be able to manage these cases appropriately in clinical practice.

\section{Case Report}

The patient was a 40 year old male who presented with progressive, gradual onset breathlessness, exertional fatigue and generalized nonspecific weakness for the last 6 months. There was no history of angina, syncope, palpitations, edema or any overt neurological deficit. He had no past history of coronary artery disease, diabetes or hypertension, nor was he receiving any medications. Clinical examination revealed mild cardiomegaly and a grade III/VI ejection systolic murmur which increased with dynamic auscultation (on performing the Valsalva maneuver and on standing). The 12 lead ECG demonstrated left ventricular hypertrophy (LVH) by voltage criteria and a LV systolic strain pattern. A trans-thoracic 2D echocardiography confirmed obstructive HCM as evidenced by asymmetric septal hypertrophy, systolic anterior motion of mitral valve and a gradient across the left ventricular outflow tract. The resting peak gradient was $24 \mathrm{~mm} \mathrm{Hg}$ which increased to 50 $\mathrm{mm} \mathrm{Hg}$, after administration of sublingual nitroglycerin, confirming the presence of dynamic LV outflow tract obstruction characteristic of obstructive HCM. In addition, there was severe mitral regurgitation with a posteriorly directed jet. Oral beta-blocker therapy (Metoprolol $50 \mathrm{mg} \mathrm{BD}$ ) was initiated and the patient advised for reassessment of symptoms and the gradient at 4 weeks.

However the patient presented again, 2 weeks later with progressively increasing bilateral upper limb weakness and difficulty in masticating food. There was a characteristic diurnal variation of symptoms, with worsening being noted towards the evening hours. Clinical examination revealed bilateral ptosis with poor arm hanging time, suggesting a diagnosis of myasthenia gravis. Anti-cholinesterase antibody assay was positive and Electromyography was suggestive of Myasthenia gravis. The cardiac and echocardiographic findings were unchanged. Slight mediastinal widening was noted on the X-ray chest while a CT chest revealed an anterior mediastinal mass suggestive of thymoma (Figure 1). The patient underwent trans-sternal thymectomy with extraction of a thymic mass measuring approximately 70 grams without any procedural complications (Figure 2). Histopathology revealed a minimally invasive Thymoma (WHO Type B2).

The patient was subsequently discharged on Pyridostigmine, prednisoslone and oral Metoprolol with a plan for alcohol septal ablation symptoms related to HCM persist. He remains asymptomatic at the last follow up of 3 months.

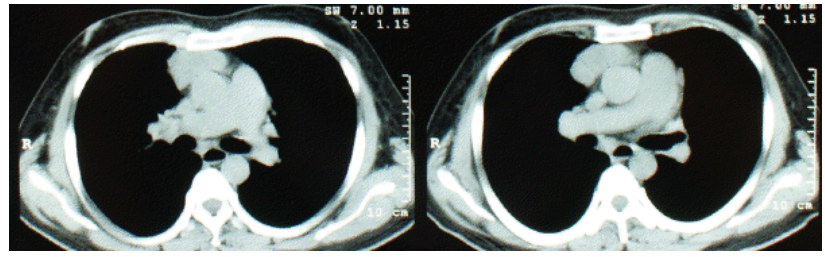

Figure 1: CT chest demonstrating an anterior mediastinal mass suggestive of thymoma.

*Corresponding author: Aditya Kapoor, Sanjay Gandhi PGIMS, Lucknow 226014 India, Tel: 0091522 2494220; Fax: 910522 2668073; E-mail: akapoor65@gmail.com

Received January 17, 2014; Accepted February 20, 2014; Published February 22, 2014

Citation: Nadig S, Kapoor A, Agrawal R, Mishra A (2014) Unmasking of Occult Myasthenia Gravis in Hypertrophic Cardiomyopathy by Beta Blockers. J Clin Case Rep 4: 350. doi:10.4172/2165-7920.1000350

Copyright: $\odot 2014$ Nadig S, et al. This is an open-access article distributed under the terms of the Creative Commons Attribution License, which permits unrestricted use, distribution, and reproduction in any medium, provided the original author and source are credited. 


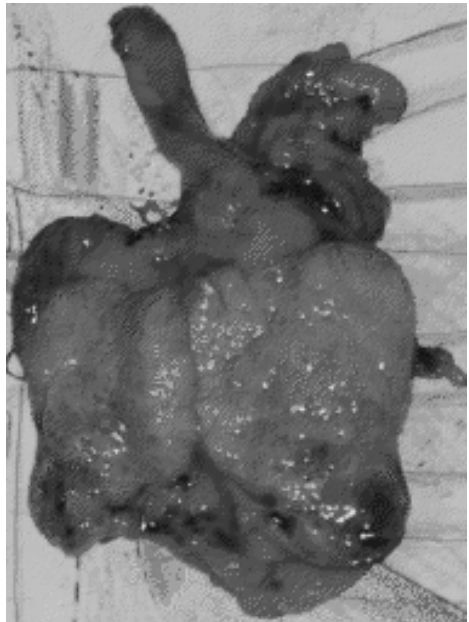

Figure 2: Trans-sternal thymectomy with extraction of a thymic mass measuring approximately 70 grams.

\section{Discussion}

Hypertrophic cardiomyopathy is a relatively common cardiac disorder, occurring in 1 out of 500 people, and is caused by various mutations in genes encoding for different cardiac sarcomeric proteins [1]. The characteristic feature is asymmetrical myocardial hypertrophy involving the interventricular septum, resulting in an obstruction of flow through the left ventricular (LV) outflow tract, as also seen in our case. Oral beta blockers are an important mainstay in the pharmacological management of these patients. Due to their heart rate lowering effects, these drugs reduce the myocardial oxygen consumption and resultant myocardial ischemia. Beta blockers also reduce the gradient across LV outflow tract due to their negative inotropic effect and improve the diastolic dysfunction and LV compliance.

Myasthenia gravis is an autoimmune disorder of neuromuscular transmission, which usually presents as oculobulbar and proximal muscle weakness and easy fatigability following repetitive effort. Our patient possibly had pre-existent latent myasthenia gravis, since he did complain of easy fatigability and weakness. However patients with obstructive HCM also often present with shortness of breath and exertional fatigue due to the reduced LV compliance, diastolic dysfunction and inability to adequately increase the cardiac output in response to activity and exercise. Hence the patient's symptoms of fatigue and exertional weakness were interpreted as part of the symptomatology of HCM. Moreover at that time, he did not have any overt clinical signs of myasthenia gravis. An underlying thymoma, as occurred in our case, is the underlying etiology in about $10-15 \%$ cases of myasthenia gravis [2].

Following institution of oral beta blocker therapy for HCM in our case, there was a rapid unmasking of the classical symptoms and signs of myasthenia gravis. Various pharmacologic agents including beta blockers [3,4] calcium channel blockers, [5] anti-arrhythmics, [6] inhalational anesthetic agents [7] and antibiotics [8] can affect neuromuscular transmission, potentially precipitating or unmasking underlying signs and symptoms of myasthenia gravis.

Symptoms like fatigue, generalized weakness and shortness of breath which are common among patients with cardiac diseases are also frequent in patients with myasthenia gravis. Hence it is not unusual to attribute these symptoms to the associated cardiac condition rather than myasthenia gravis, as happened in our case [9]. Our case also highlights the fact that commonly used cardiac medications (in this particular case, beta blocker Metoprolol) can potentially unmask or exacerbate symptoms and signs of myasthenia gravis in patients with no obvious known defects of neuromuscular transmission. Detailed clinical examination led to a clinical diagnosis of underlying myasthenia gravis which was secondary to a thymoma.

Cardiac involvement in myasthenia gravis although uncommon, may include asymptomatic ECG changes, cardiac arrhythmias, myocarditis, conduction disorders and rarely reversible heart failure (stress induced cardiomyopathy), usually in the setting of myasthenic crisis [10,11]. The association of HCM with myasthenia gravis is extremely rare. Apart from the current case, there is only one previously reported instance; and that was a case of non-obstructive HCM who had known pre-existing myasthenia gravis [9]. Our case in contrast, had obstructive HCM and was not a known case of myasthenia gravis; unmasking of muscle weakness and appearance of typical signs of myasthenia gravis appeared only after institution of beta blocker therapy.

\section{Conclusion}

It is important for physicians to be aware of such unusual associations to be able to appropriately manage such cases in clinical practice. Frequently used cardiac medications can potentially unmask or exacerbate symptoms and signs of myasthenia gravis in patients with no obvious known defects of neuromuscular transmission. A high index of suspicion and detailed clinical examination can be helpful in avoiding mis-diagnosis and improving patient related outcomes.

\section{References}

1. Maron BJ (2002) Cardiology patient pages. Hypertrophic cardiomyopathy. Circulation 106: 2419-2421.

2. Romi F, Skeie GO, Aarli JA, Gilhus NE (2000) Muscle autoantibodies in subgroups of myasthenia gravis patients. J Neurol 247: 369-375.

3. Herishanu Y, Rosenberg P (1975) Letter: beta-Blockers and myasthenia gravis. Ann Intern Med 83: 834-835.

4. Jonkers I, Swerup C, Pirskanen R, Bjelak S, Matell G (1996) Acute effects of intravenous injection of beta-adrenoreceptor- and calcium channel at antagonists and agonists in myasthenia gravis. Muscle Nerve 19: 959-965.

5. Krendel DA, Hopkins LC (1986) Adverse effect of verapamil in a patient with the Lambert-Eaton syndrome. Muscle Nerve 9: 519-522.

6. Shy ME, Lange DJ, Howard JF (1985) Quinidine exacerbating myasthenia gravis: a case report and intracellular recordings. Annals of Neurology 18:120.

7. Baraka A, Afifi A, Muallem M, Kachachi T, Frayha F (1971) Neuromuscular effects of halothane, suxamethonium and tubocurarine in a myasthenic undergoing thymectomy. Br J Anaesth 43: 91-95.

8. Dretchen KL, Gergis SD, Sokoll MD, Long JP (1972) Effect of various antibiotics on neuromuscular transmission. Eur J Pharmacol 18: 201-203.

9. Drolet B, Gabra G, Simard C, Noel B, Poirier P (2009) Verapamil-associated cardiogenic shock in a 71-year-old man with myasthenia gravis: a case report. J Med Case Rep 3: 8219.

10. Bansal V, Kansal MM, Rowin J (2011) Broken heart syndrome in myasthenia gravis. Muscle Nerve 44: 990-993.

11. Bijulal S, Harikrishnan S, Namboodiri N, Ajitkumar VK, Gupta D, et al. (2009) Tako-tsubo cardiomyopathy in a patient with myasthenia gravis crisis: a rare clinical association. BMJ Case Rep 2009. 